\title{
MicroRNA-375 suppresses esophageal cancer cell growth and invasion by repressing metadherin expression
}

\author{
CHANGMEI HU ${ }^{1}$, LIANG LV ${ }^{1}$, JIE PENG ${ }^{2}$, DELIANG LIU ${ }^{1}$, \\ XUEHONG WANG ${ }^{1}$, YUQIAN ZHOU ${ }^{1}$ and JIRONG HUO ${ }^{1}$ \\ ${ }^{1}$ Department of Gastroenterology, The Second Xiangya Hospital of Central South University, Changsha, Hunan 410011; \\ ${ }^{2}$ Department of Haematology, Xiangya Hospital of Central South University, Changsha, Hunan 410078, P.R. China
}

Received February 20, 2016; Accepted February 3, 2017

DOI: $10.3892 / \mathrm{ol} .2017 .6098$

\begin{abstract}
Accumulating evidence indicates that aberrant expression of microRNAs is involved in tumorigenesis, tumor progression and response to therapy. MicroRNA-375 (miR-375) is an important cancer-associated RNA that is downregulated in multiple types of cancer. In the present study, the potential effects of and underlying molecular mechanism for miR-375 in esophageal cancer were investigated. The expression of miR-375 in paired esophageal squamous cell carcinoma (ESCC) and non-tumor tissues from 10 patients was quantified using the reverse transcription-quantitative polymerase chain reaction. The miR-375 levels in the ESCC cell line EC109 and a normal esophageal epithelial cell line, Het-1A, were also detected. The effect of miR-375 on ESCC cell growth and invasion was determined using Cell Counting kit-8, flow cytometry and invasion assays. A luciferase assay was conducted for target identification. The results of the present study revealed that miR-375 was downregulated in ESCC tumor tissue and EC109 cells compared with normal tissue and Het-1A cells $(\mathrm{P}<0.01)$. Overexpression of miR-375 inhibited EC109 cell growth and invasion, and induced cell cycle arrest. In addition, metadherin (MTDH) was demonstrated to be a direct target of miR-375 $(\mathrm{P}<0.01)$. The overexpression of miR-375 downregulated MTDH $(\mathrm{P}<0.01)$, cyclin D1 $(\mathrm{P}<0.05)$ and vascular endothelial growth factor $(\mathrm{P}<0.01)$ expression, while upregulating epithelial cadherin $(\mathrm{P}<0.01)$ expression, which may account for its effect on ESCC cell proliferation and invasion. The results of the present study suggest that the miR-375/MTDH axis represents a target for the treatment of ESCC.
\end{abstract}

Correspondence to: Dr Jirong Huo, Department of Gastroenterology, The Second Xiangya Hospital of Central South University, 139 Middle Renmin Road, Changsha, Hunan 410011, P.R. China

E-mail: 13808471870@163.com

Key words: esophageal squamous cell carcinoma, metadherin, miR-375, cell proliferation, cell invasion

\section{Introduction}

Esophageal cancer, which includes esophageal squamous cell carcinoma (ESCC) and adenocarcinoma of the esophagus (EADC), is one of the most prevalent types of malignant tumor worldwide (1). Esophageal cancer occupies the sixth position among malignant tumors worldwide with regard to mortality and ranks fourth in China (2). ESCC is the most predominant esophageal cancer type in Asia (3). Surgery, radiotherapy and chemotherapy are the typical treatments for ESCC. Due to a lack of effective clinical diagnosis methods for ESCC, it is often diagnosed in the later stages of the disease. The 5-year overall survival rate of ESCC is between 20 and $30 \%$ (4). Investigating the mechanism of ESCC progression is essential to improve disease diagnosis, treatment and prognosis.

MicroRNAs (miRNAs) are a large class of highly conserved small non-coding RNAs that negatively regulate gene expression through binding the 3 ' untranslated region (3'-UTR) of target mRNAs, causing mRNA degradation and therefore repression of translation (5). In total $>1,000$ miRNAs have been identified in the human genome, which may regulate the expression of $<30 \%$ of all protein-coding genes (6). Numerous studies have identified that aberrant expression of miRNAs is associated with tumorigenesis, tumor progression and response to therapy (7). miR-375 was initially characterized as a pancreatic islet-specific miRNA, and it regulates glucose homeostasis and glucose-induced insulin secretion $(8,9)$. Further miRNA expression profiling studies revealed that miR-375 is downregulated in multiple types of cancer and acts as a tumor suppressor. For example, miR-375 was demonstrated to repress gastric cancer growth by targeting Janus kinase 2 (10). It has also been reported that miR-375 inhibits the proliferation and invasion of human squamous cervical cancer cells by repressing transcription factor SP1 (11). In ESCC, miR-375 is one of the most frequently downregulated miRNAs (10). However, the tumor suppression mechanism of miR-375 in ESCC has not been fully elucidated.

In the present study, it was identified that miR-375 is downregulated in ESCC tumor tissue and the ESCC cell line EC109. It was also identified that miR-375 acts as a tumor suppressor in EC109 cells, and that metadherin (MTDH) is a direct target. The effect of miR-375 on ESCC cell proliferation and invasive 
ability may be mediated by the downregulation of MTDH, suggesting that MTDH is a potential therapeutic target for ESCC therapy.

\section{Materials and methods}

Patients and sample collection. A total of 10 patients who had been diagnosed with ESCC were recruited to participate in the present study between May 2013 and October 2013 at Xiangya Hospital of Central South University (Changsha, China). All patients underwent esophageal cancer resection prior to administration of chemotherapy; following resection, ESCC and corresponding non-cancerous tissues were collected immediately and validated by pathologists. All patients provided written informed consent. All experiments were performed with the approval of the Ethics Committee of the Xiangya Hospital of Central South University.

Cell lines and cell culture. The ESCC cell line EC109 and the human immortalized normal esophageal epithelial cell line Het-1A were purchased from Changsha Yingrun Biotechnology Co. Ltd. (Changsha, China). Cells were cultured in Dulbecco's modified Eagle's medium (DMEM; Gibco; Thermo Fisher Scientific, Inc., Waltham, MA, USA) and supplemented with $10 \%$ fetal bovine serum (FBS; Gibco; Thermo Fisher Scientific, Inc.), $100 \mathrm{U} / \mathrm{ml}$ penicillin and $100 \mu \mathrm{g} / \mathrm{ml}$ streptomycin in a humidified incubator with $5 \% \mathrm{CO}_{2}$ at $37^{\circ} \mathrm{C}$.

Reverse transcription-quantitative polymerase chain reaction (RT-qPCR) analysis. Total miRNA was extracted using an miRNeasy kit (Qiagen GmbH, Hilden, Germany), according to the manufacturer's protocol. RNA was converted into cDNA using an miScriptRT kit (Qiagen $\mathrm{GmbH}$ ), according to the manufacturer's protocol. qPCR was performed using an miScript SYBR Green PCR kit (Qiagen $\mathrm{GmbH}$ ) in an ABI 7,500 Real-Time PCR System (Applied Biosystems; Thermo Fisher Scientific, Inc.). PCR reactions were run at $95^{\circ} \mathrm{C}$ for $10 \mathrm{~min}$, followed by 40 cycles of $95^{\circ} \mathrm{C}$ for $15 \mathrm{sec}$ and $60^{\circ} \mathrm{C}$ for $1 \mathrm{~min}$. The detected expression of miRNAs was normalized using small nuclear RNA U6 (U6) as an endogenous control. miR-375 and U6 primers (miR-375 forward, 5'-gcagatttgttc gttcggctc-3' and reverse, 5'-gtgcagggtccgaggt-3'; and U6 forward, 5'-ctcgcttcggcagcaca-3' and reverse 5'-aacgcttca cgaatttgcgt-3') were purchased from Changsha Yingrun Biotechnology Co. Ltd. The RT-qPCR assays were performed in triplicate and the change in expression level was calculated using the $2^{-\Delta \Delta C q}$ method (ABI 7500 Software v2.0.1; Applied Biosystems; Thermo Fisher Scientific, Inc.) (12).

Proliferation assays. A Cell Counting kit-8 (CCK-8; Beyotime Institute of Biotechnology, Haimen, China) assay was used to measure cell proliferation. A total of $2 \times 10^{4}$ cells/well were plated in 96-well plates and cultured under the aforementioned conditions. Cell proliferation was monitored each day. To allow cell counting, $10 \mu \mathrm{l}$ CCK- 8 reagent was added to each well and the plates were incubated at $37^{\circ} \mathrm{C}$ for $2 \mathrm{~h}$. The absorbance values were subsequently measured at $450 \mathrm{~nm}$ using a microplate reader (Bio-Rad Laboratories, Inc., Hercules, CA, USA). Each experiment was performed in triplicate.
Transfection of miR-375 mimic. The miR-375 mimic (sense, 5'-uuuguucguucggcucgeguga-3' and antisense, 5'-acgegagcc gaacgaacaaauu-3') and a negative control (NC; sense, 5'-uuc uccgaacgugucacgutt-3' and antisense, 5'-acgugacacguucgg aga att-3') were purchased from GenePharm (Shanghai,China). Cells were plated at $3 \times 10^{5}$ per well in 6 -well plates and cultured for $24 \mathrm{~h}$. The cells were subsequently transfected with miR-375 mimic or NC RNA, to a final concentration of $75 \mathrm{nM}$, using Lipofectamine 2000 (Invitrogen; Thermo Fisher Scientific, Inc.) and FBS-free Opti-MEM medium (Gibco; Thermo Fisher Scientific, Inc.). After $6 \mathrm{~h}$, the medium was replaced with DMEM containing 10\% FBS.

Flow cytometric analysis of apoptosis. Apoptotic cells were measured using an Annexin V-fluorescein isothiocyanate (FITC) Apoptosis Detection kit (Nanjing KeyGen Biotech Co., Ltd, Nanjing, China). The cell samples were harvested using $0.25 \%$ EDTA-free trypsin after $48 \mathrm{~h}$ of incubation, washed twice with ice-cold PBS and resuspended in $500 \mu 1$ binding buffer. Cells were incubated with $5 \mu 1$ annexin V-FITC-labeled specific antibodies (from the apoptosis detection kit) and $5 \mu 1$ propidium iodide (PI) for between 15 and $20 \mathrm{~min}$ in the dark, and detected using a FACSAria flow cytometer and FASCDiva 4.1 software (BD Biosciences, Franklin Lakes, NJ, USA). Each experiment was performed in triplicate.

Cell cycle assays. Following transfection with miR-375 mimic or NC, the cell samples were harvested as aforementioned and fixed with $75 \%$ ethanol at $4^{\circ} \mathrm{C}$ overnight. The cells were washed in ice-cold PBS and treated with RNase I and Triton-X-100, followed by PI staining for $30 \mathrm{~min}$ in the dark. Finally, cells were washed and resuspended in PBS. Cell cycle distributions were detected using a BD Biosciences FACSAria flow cytometer.

Invasion assays. The invasiveness of cells was determined using a Transwell system [24-well plate, $8 \mu \mathrm{m}$ pore polyethylene terephthalate (PET) membrane; Corning Incorporated, Corning, NY, USA]. The PET membrane was coated with Matrigel (dilution, 1:5; BD Biosciences) in FBS-free DMEM. Following transfection, the cell samples were harvested as aforementioned and resuspended in FBS-free DMEM, for seeding in the upper chamber. DMEM containing $10 \%$ FBS was added to the lower chamber. Following incubation at $37^{\circ} \mathrm{C}$ for $24 \mathrm{~h}$, cells that appeared on the lower surface of the membrane were fixed with methanol for $15 \mathrm{~min}$ at $4^{\circ} \mathrm{C}$, then stained with $0.1 \%$ crystal violet solution and counted under a light microscope.

Construction of vector and luciferase reporter assay. The 3'-UTR of the MTDH gene (positions 1454-1461) was predicted to be complementary to the sequence of miR-375 according to an analysis using the miRNA target gene prediction database TargetScan 7.1 (13). Fragments of sequence from wild-type and mutant MTDH 3'-UTR were amplified by PCR and cloned into a pYr-MirTarget luciferase vector (Changsha Yingrun Biotechnology Co. Ltd.), designated pYr-MTDH-3U and PYr-MTDH-3Umt, respectively. The primers for the wild-type 3'-UTR, containing XhoI and NotI restriction sites, 
were as follows: Forward, 5'-ccgctcgagtgtggcgccaagaacgaa-3', and reverse, 5'-aaagcggccgcatactgccatgttcctgaa-3'. The primers for the mutant $3^{\prime}$-UTR were as follows: Forward, 5'-ttgtcagac taggaaagctaaaaaaatggttttgctgaa-3'; reverse, 5'-ttcagcaaaactaaa accatttttttagctttcctagtctgacaa-3'. HEK293 cells (American Type Culture Collection, Manassas, VA, USA) were plated into a 96-well plate and co-transfected with $100 \mathrm{ng}$ pYr-MTDH-3U or pYr-MTDH-3Umt and miR-375 mimic or NC (75 nM) using Lipofectamine 2000. Luciferase activity was determined using a Dual-Luciferase Reporter Assay kit (Promega Corporation, Madison, WI, USA), according to the manufacturer's protocol. Each experiment was performed in triplicate.

Western blot analysis. Cells were harvested and homogenized with lysis buffer (Beyotime Institute of Biotechnology) $48 \mathrm{~h}$ post-transfection. Protein concentrations were measured using a BCA Protein Assay kit (Beyotime Institute of Biotechnology). Proteins (between 30 and $50 \mu \mathrm{g}$ ) were separated by SDS-PAGE (10\% gel) and transferred onto nitrocellulose membranes (EMD Millipore, Billerica, MA, USA). Subsequent to blocking with 5\% skimmed milk powder at room temperature for $2 \mathrm{~h}$, the membranes were incubated with primary antibodies specific to MTDH (catalog no., 14065S), vascular endothelial growth factor C (VEGF-C; catalog no., 2445S), cyclin D1 (catalog no., 2978T), epithelial (E-) cadherin (catalog no., 3195S) and $\beta$-actin (catalog no., 3700S) (all dilution, 1:1,000; Cell Signaling Technology, Inc., Danvers, MA, USA). The membranes were then incubated with corresponding horseradish peroxidase-conjugated secondary antibodies (goat anti-mouse secondary antibody for $\beta$-actin; dilution, 1:5,000; catalog no., sc-2005 or mouse anti-rabbit secondary antibody for MTDH, VEGF-C, cyclin D1 and E-cadherin; catalog no., sc-2357; Santa Cruz Biotechnology, Inc., Dallas, TX, USA). The resulting immunoblots were quantified using ImageJ2x software (National Institutes of Health, Bethesda, MD, USA).

Statistical analysis. Data were expressed as the mean \pm standard deviation from at least three independent experiments and statistically analyzed using a one-way analysis of variance test followed by Tukey's multiple comparison test or an independent sample t-test. All statistics were performed using SPSS software (version 17.0; SPSS, Inc., Chicago, IL, USA). P $<0.05$ was considered to indicate a statistically significant difference, and $\mathrm{P}<0.01$ was considered to indicate a highly significant difference.

\section{Results}

miR-375 is downregulated in clinical esophageal cancer tissues and the ESCC cell line EC109. The level of miR-375 in clinical esophageal carcinoma and adjacent non-tumor tissues from 10 ESCC patients were measured using RT-qPCR. The results revealed that the miR-375 level was significantly decreased in tumor tissues compared with in non-tumor tissues (Fig. 1A; $\mathrm{P}<0.01$ ). Additionally, miR-375 expression in the ESCC cell line EC109 was significantly downregulated compared with that in Het-1A, a normal human esophageal endothelial cell line (Fig. 1B, P<0.01). These results suggested that downregulation of miR-375 may be associated with the pathogenesis of esophageal cancer.

miR-375 suppresses cell growth and induces cell cycle arrest in EC109 cells. To explore the roles of miR-375 in esophageal cancer progression, EC109 cells were transfected with miR-375 mimic or a NC. Cell proliferation rate, the proportion of apoptotic cells and cell cycle distribution were measured. Overexpression of miR-375 by miR-375 mimic transfection was confirmed using RT-qPCR (Fig. 2A). miR-375 overexpression significantly inhibited the proliferation of EC109 cells (Fig. 2B). Flow cytometric analysis revealed that overexpression of miR-375 modulated the cell cycle by inducing G1 arrest (Fig. 2C). No significant difference between the NC and miR-375 mimic group in the rate of apoptosis was identified (Fig. 2D). These results suggested that the overexpression of miR-375 inhibited cell proliferation through triggering cell cycle arrest rather than apoptosis.

miR-375 suppresses invasion in EC109 cells. EC109 cells transfected with miR-375 mimic or an NC were analyzed using Transwell invasion assays. As presented in Fig. 3, overexpression of miR-375 repressed the invasive ability of EC109 cells by $\sim 67 \%$ compared with the $\mathrm{NC}$ group $(\mathrm{P}<0.01)$.

$M T D H$ is a direct target gene of $m i R-375$. To further explore the molecular mechanism of miR-375 in ESCC progression, computational algorithms were applied (using TargetScan) to predict potential target mRNAs of miR-375. It was identified that the MTDH mRNA 3'-UTR contained highly conserved binding sites for miR-375 (Fig. 4A). Dual-luciferase assays were performed to confirm this prediction. HEK293 cells were co-transfected with pYr-MTDH-3U and miR-375 mimic. The assessment of luciferase activity demonstrated that miR-375 mimic significantly decreased the luciferase activity of pYr-MTDH-3U compared with the NC (Fig. 4B; $\mathrm{P}<0.01)$. No significant difference in the luciferase activity of pYr-MTDH-3Umt was identified (Fig. 4C). miR-375 mimic downregulated the expression of MTDH in EC109 cells $(\mathrm{P}<0.01)$, further confirming that MTDH is a target gene of miR-375. The expression of the MTDH downstream protein VEGF-C $(\mathrm{P}<0.01)$ and cell cycle regulator cyclin $\mathrm{D} 1(\mathrm{P}<0.05)$ were decreased by miR-375 mimic (Fig. 4D). By contrast, miR-375 mimic significantly upregulated expression of the epithelial marker E-cadherin (Fig. 4D; P<0.01), which may inhibit tumor cell epithelial-mesenchymal transition (EMT) and invasion (14).

\section{Discussion}

ESCC has a poor prognosis and high mortality rate (2). Cancer metastasis is the primary cause of cancer-associated mortality; therefore, preventing metastasis may be an effective strategy for cancer therapy. A number of studies have provided novel insights into the underlying molecular mechanism of regulation of gene expression by miRNAs. It has been reported that the aberrant expression of miRNA is associated with tumorigenesis, tumor progression, cancer prognoses and response to therapy $(7,15,16)$. In the present study, it was demonstrated that miR-375 was downregulated in ESCC tumor tissue samples 

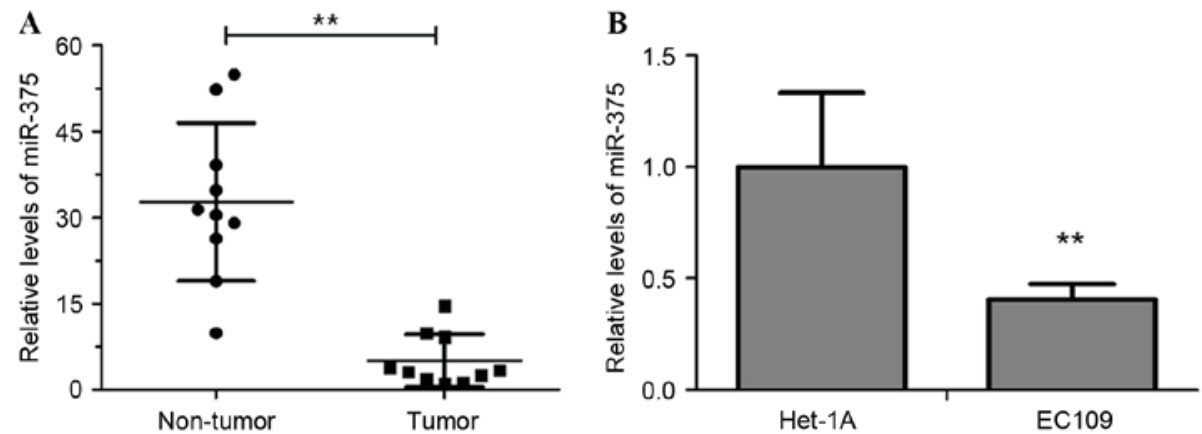

Figure 1. miR-375 is downregulated in human ESCC. (A) RT-qPCR analysis of miR-375 expression in tumor and paired adjacent non-tumor tissues of 10 ESCC patients. (B) RT-qPCR analysis of miR-375 expression in the ESCC cell line EC109 and the normal human esophageal endothelial cell line Het-1A. Experiments were performed in triplicate and data are expressed as the mean \pm standard deviation. ${ }^{* *} \mathrm{P}<0.01$. miR-375, microRNA-375; RT-qPCR, reverse transcription-quantitative polymerase chain reaction; ESCC, esophageal squamous cell carcinoma.
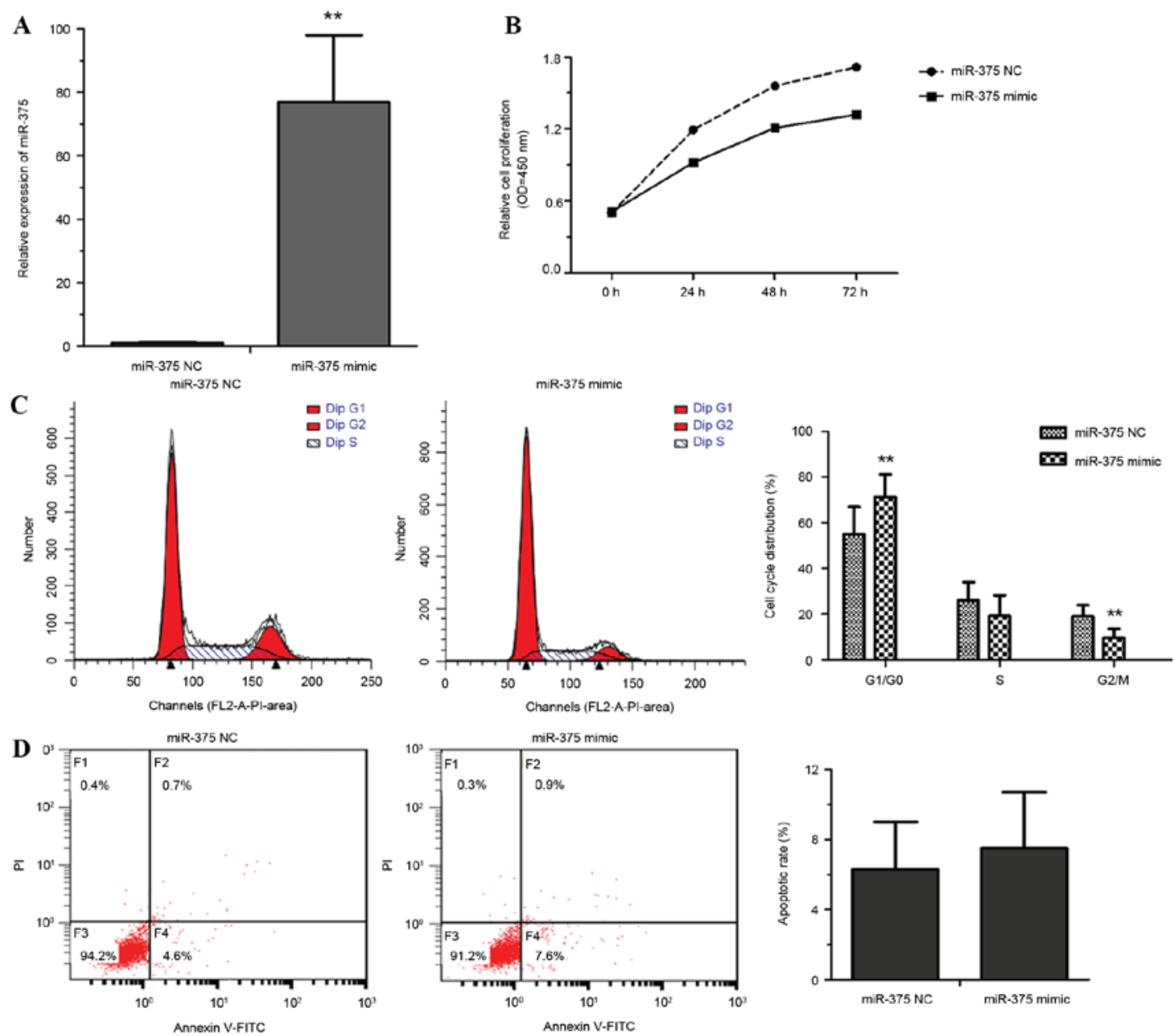

Figure 2. miR-375 suppresses cell growth and induces cell cycle arrest in EC109 cells. (A) Reverse transcription-quantitative polymerase chain reaction analysis of miR-375 expression in miR-375 mimic- or NC-transfected EC109 cells. (B) The growth rate of miR-375 mimic- or NC-transfected EC109 cells was measured using a Cell-Counting kit 8 assay. (C) Flow cytometric analysis of the cell cycle distribution of miR-375 mimic- or NC-transfected EC109 cells. (D) Flow cytometric analysis of apoptosis revealed no difference in apoptosis rates between miR-375 mimic- and NC-transfected EC109 cells. Experiments were performed in triplicate and data are expressed as the mean \pm standard deviation. " $\mathrm{P}<0.05$ vs. NC. miR-375, microRNA-375; NC, negative control; OD, optical density; PI, propidium iodide; FITC, fluorescein isothiocyanate.

and the ESCC cell line EC109 compared with non-tumor tissues and Het-1A, a normal esophageal endothelial cell line, respectively. Furthermore, overexpression of miR-375 in EC109 cells was demonstrated to inhibit cell proliferation and invasion, and induce cell cycle arrest. MTDH was identified as a target gene of miR-375; the results of the present study indicate that miR-375 acts as a tumor suppressor in EC109 cells by modulating the expression of MTDH.

Previous studies have identified several miRNAs suitable for use in distinguishing ESCC from non-tumor tissue. 

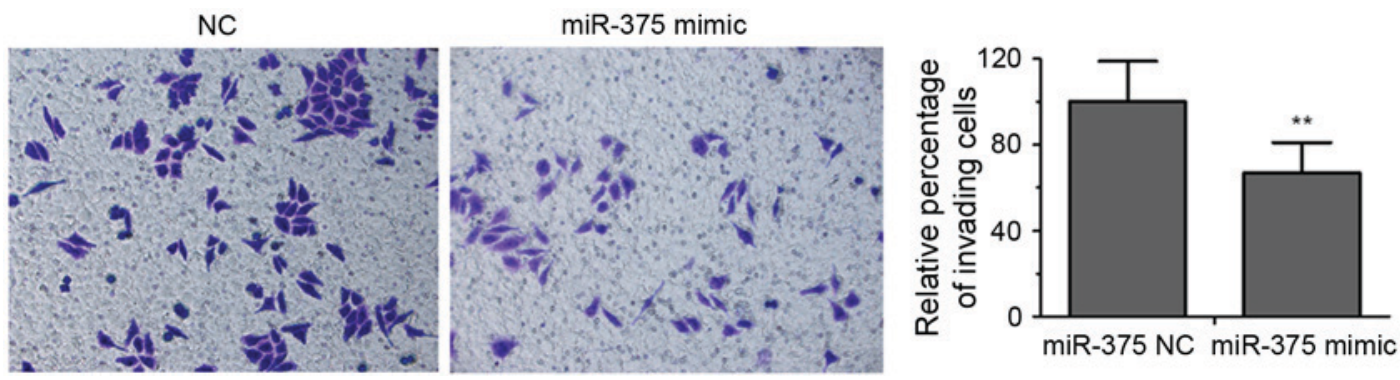

Figure 3. Overexpression of miR-375 suppresses EC109 cell invasion. Photomicrographs of Transwell membranes indicating the cells that crossed the $8 \mu \mathrm{m}$ pore membrane with Matrigel (magnification, x200). Quantitative analysis of relative numbers of invaded cells is presented in the right panel. Experiments were performed in triplicate and data are expressed as the mean \pm standard deviation. ${ }^{* *} \mathrm{P}<0.01$ vs. NC. miR-375, microRNA-375; NC, negative control.

A

Position 1454-1461 of MTDH 3'UTR

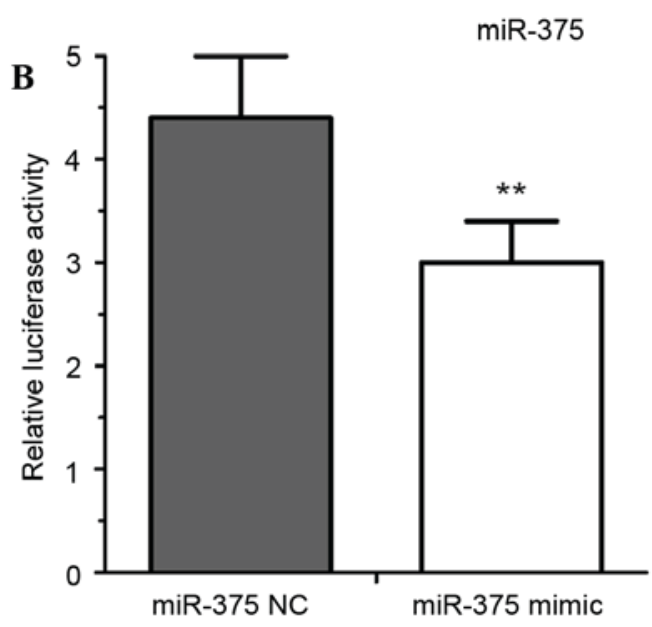

D

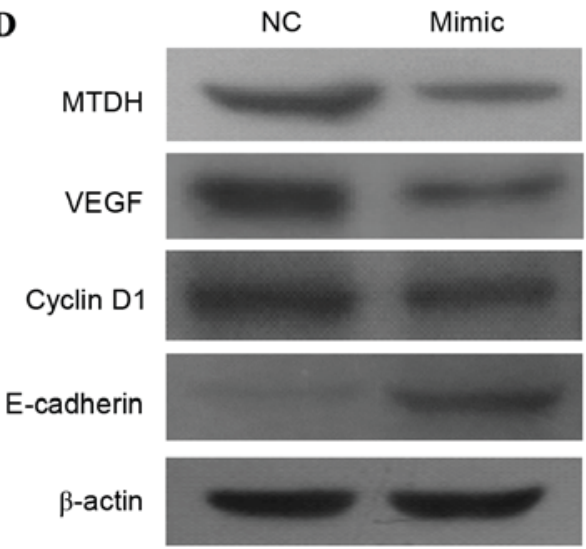

5'....ACUAGGAAAGCUAAACGAACAAA....

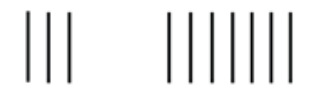

3' AgugcgcucggcuUgCuUguUU

C
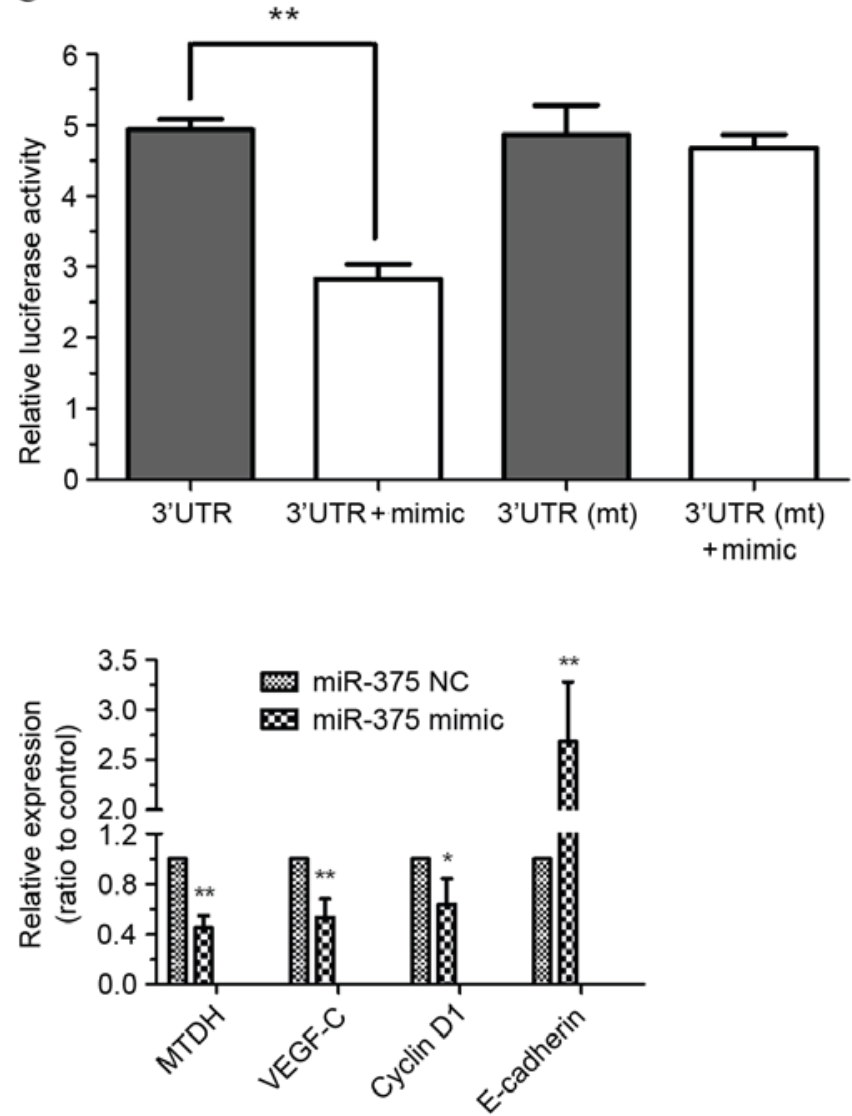

Figure 4. MTDH is a direct target gene of miR-375. (A) The 3'-UTR of MTDH mRNA includes a highly conserved binding site for miR-375. (B) HEK293 cells were co-transfected with pYr-MTDH-3U and an miR-375 mimic or NC. At $24 \mathrm{~h}$ after transfection, luciferase activity was detected. (C) Dual-luciferase assays demonstrated miR-375 mimic-mediated repression of pYr-MTDH-3U luciferase; however, no significant difference from pYr-MTDH-3Umt luciferase was identified. (D) miR-375 modulated the expression of MTDH, cyclin D1, VEGF-C and E-cadherin in EC109 cells. $\beta$-Actin served as a loading control. Experiments were performed in triplicate and data are expressed as the mean \pm standard deviation, ${ }^{* *} \mathrm{P}<0.01$ vs. NC. MTDH, metadherin; miR-375, microRNA-375; 3'-UTR, 3'-untranslated region; NC, negative control; mt, mutated; VEGF-C, vascular endothelial growth factor.

Guo et al (4) reported four miRNAs (miR-100, -99a, -29c and -140) that were downregulated and three miRNAs (miR-25, -424 and -151) that were upregulated in ESCC. Yan et al (17) confirmed that miR-340 is significantly downregulated in ESCC and inhibits ESCC cell growth and invasion by targeting phosphoserine aminotransferase 1. miR-204 was demonstrated to repress EC109 EMT and invasion by targeting box protein M1 (18). In addition, the circulating levels of miR-21 and miR-375 in patients with ESCC may act as reliable prognostic markers for ESCC (19). 
It has been demonstrated that miR-375 predominantly serves as a tumor suppressor, particularly in tumors of the digestive tract (10). In hepatocellular carcinoma, ectopic expression of miR-375 decreases cell growth and invasion, and induces G1 arrest and apoptosis (20). miR-375 has been reported as one of the most frequently downregulated miRNAs in esophageal cancer (21). Similarly, in the present study, it was demonstrated that miR-375 was significantly decreased in ESCC tumor tissue and the ESCC line EC109. Overexpression of miR-375 in EC109 repressed cell growth and invasion; however, it did not induce apoptosis in EC109 cells. miR-375-induced G1 cell cycle arrest was the primary cause for the inhibition of EC109 cell proliferation.

Although several studies have explored the role of miRNAs in esophageal cancer, the identified potential targets require further confirmation. For example, overexpression of miR-145, $-133 \mathrm{a}$ or $-133 \mathrm{~b}$ in ESCC may inhibit cell proliferation and invasion; they all may repress the expression of Fascin homolog 1 (22). miR-375 inhibits ESCC cell proliferation through targeting insulin-like growth factor receptor 1 and 3-phosphoinositide-dependent protein kinase-1, which are involved in the potentially carcinogenic phosphoinositide 3-kinase (PI3K)/protein kinase B (AKT) signaling pathway (10). To investigate the mechanism of miR-375 function in ESCC cells, computational algorithms were used to predict possible target mRNAs of miR-375. The intersection of algorithms and a luciferase reporter assay indicated that MTDH was a target gene of miR-375. MTDH was originally identified as an oncogene (23) upregulated in numerous types of malignancy, including breast cancer, neuroblastoma, non-small cell lung cancer and cancers of the digestive tract (24-26). A number of studies have demonstrated that the upregulation of MTDH promotes tumor progression through modulating signaling pathways including the PI3K/AKT, Wnt/ $\beta$-catenin and nuclear factor $\mathrm{\kappa B}$ signaling pathways (24). In the present study, overexpression of miR-375 in EC109 cell significantly decreased the expression of MTDH.

To further investigate the mechanism of miR-375 on tumor growth and invasiveness, the expression levels of VEGF-C, E-cadherin and cyclin D1 were examined. The results demonstrated that cyclin D1 and VEGF-C were downregulated, whereas E-cadherin was upregulated in miR-375-overexpressing EC109 cells. The majority of tumors and metastases begin as avascular masses limited in size (27), therefore VEGF-C, an inducer of angiogenesis, is a critical factor for tumor growth and development (28). It has been confirmed that MTDH can directly upregulate VEGF-C expression by enhancing VEGF-C promoter activity and activating the $\mathrm{PI} 3 \mathrm{~K} / \mathrm{AKT}$ signaling pathway $(29,30)$. Cyclin D1 is essential for G1 phase progression and is frequently overexpressed in a broad range of human cancers (31). The overexpression of MTDH was observed in chronic lymphocytic leukemia and was identified to be associated with the Rai stage classification. Inhibition of MTDH expression resulted in decreased levels of cyclin D1 via suppression of the Wnt $/ \beta$-catenin signaling pathway (32). The epithelial marker E-cadherin serves a key role in EMT; its expression represses tumor invasion and metastasis (33). Li et al (34) have reported that overexpression of MTDH could promote EMT via downregulating E-cadherin and increasing other EMT indicators. This demonstrates the involvement of MTDH in tumorigenesis and supports the results of the present study, suggesting that downregulation of miR-375 may contribute to ESCC tumorigenesis via the resulting upregulation of MTDH.

In summary, the present study has revealed that miR-375 is underexpressed in ESCC tumor tissue samples and the ESCC cell line EC109, compared with normal tissue and cells. miR-375 has been demonstrated to act as a tumor suppressor in ESCC cells that inhibited cell proliferation and invasion by repressing the expression of its direct target MTDH, an oncogene associated with tumorigenesis in numerous types of cancer. Therefore, the miR-375/MTDH axis may represent a potential target for the treatment of ESCC.

\section{References}

1. Kamangar F, Qiao YL, Schiller JT, Dawsey SM, Fears T, Sun XD, Abnet CC, Zhao P, Taylor PR and Mark SD: Human papillomavirus serology and the risk of esophageal and gastric cancers: Results from a cohort in a high-risk region in China. Int J Cancer 119: 579-584, 2006.

2. Arnold M, Soerjomataram I, Ferlay J and Forman D: Global incidence of oesophageal cancer by histological subtype in 2012. Gut 64: 381-387, 2015.

3. Lagergren J: Oesophageal cancer in 2014: Advances in curatively intended treatment. Nat Rev Gastroenterol Hepatol 12: 74-75, 2015.

4. Guo Y, Chen Z, Zhang L, Zhou F, Shi S, Feng X, Li B, Meng X, Ma X, Luo M, et al: Distinctive microRNA profiles relating to patient survival in esophageal squamous cell carcinoma. Cancer Res 68: 26-33, 2008.

5. He L and Hannon GJ: MicroRNAs: Small RNAs with a big role in gene regulation. Nat Rev Genet 5: 522-531, 2004.

6. Chen K and Rajewsky N: The evolution of gene regulation by transcription factors and microRNAs. Nat Rev Genet 8: 93-103, 2007.

7. Fendler A, Jung M, Stephan C, Honey RJ, Stewart RJ, Pace KT, Erbersdobler A, Samaan S, Jung K and Yousef GM: miRNAs can predict prostate cancer biochemical relapse and are involved in tumor progression. Int J Oncol 39: 1183-1192, 2011.

8. Poy MN, Eliasson L, Krutzfeldt J, Kuwajima S, Ma X, Macdonald PE, Pfeffer S, Tuschl T, Rajewsky N, Rorsman P and Stoffel M: A pancreatic islet-specific microRNA regulates insulin secretion. Nature 432: 226-230, 2004.

9. van de Bunt M, Gaulton KJ, Parts L, Moran I, Johnson PR, Lindgren CM, Ferrer J, Gloyn AL and McCarthy MI: The miRNA profile of human pancreatic islets and beta-cells and relationship to type 2 diabetes pathogenesis. PLoS One 8: e55272, 2013.

10. Yan JW, Lin JS and He XX: The emerging role of miR-375 in cancer. Int J Cancer 135: 1011-1018, 2014.

11. Wang F, Li Y, Zhou J, Xu J, Peng C, Ye F, Shen Y, Lu W, Wan X and Xie X: miR-375 is down-regulated in squamous cervical cancer and inhibits cell migration and invasion via targeting transcription factor SP1. Am J Pathol 179: 2580-2588, 2011.

12. Livak KJ and Schmittgen TD: Analysis of relative gene expression data using real-time quantitative PCR and the 2(-Delta Delta C(T)) method. Methods 25: 402-408, 2001.

13. Agarwal V, Bell GW, Nam JW and Bartel DP: Predicting effective microRNA target sites in mammalian mRNAs. Elife 4, 2015.

14. Li D, Li X, Cao W, Qi Y and Yang X: Antagonism of microRNA-99a promotes cell invasion and down-regulates E-cadherin expression in pancreatic cancer cells by regulating mammalian target of rapamycin. Acta Histochem 116: 723-729, 2014.

15. Bartels CL and Tsongalis GJ: MicroRNAs: Novel biomarkers for human cancer. Clin Chem 55: 623-631, 2009.

16. Komatsu S, Ichikawa D, Takeshita H, Tsujiura M, Morimura R, Nagata H, Kosuga T, Iitaka D, Konishi H, Shiozaki A, et al: Circulating microRNAs in plasma of patients with oesophageal squamous cell carcinoma. Br J Cancer 105: 104-111, 2011.

17. Yan S, Jiang H, Fang S, Yin F, Wang Z, Jia Y, Sun X, Wu S, Jiang T and Mao A: MicroRNA-340 inhibits esophageal cancer cell growth and invasion by targeting phosphoserine aminotransferase 1. Cell Physiol Biochem 37: 375-386, 2015. 
18. Sun Y, Yu X and Bai Q: miR-204 inhibits invasion and epithelial-mesenchymal transition by targeting FOXM1 in esophageal cancer. Int J Clin Exp Pathol 8: 12775-12783, 2015.

19. Komatsu S, Ichikawa D, Takeshita H, Konishi H, Nagata $H$, Hirajima S, Kawaguchi T, Arita T, Shiozaki A, Fujiwara H, et al: Prognostic impact of circulating miR-21 and miR-375 in plasma of patients with esophageal squamous cell carcinoma. Expert Opin Biol Ther 12 (Suppl 1): S53-S59, 2012.

20. He XX, Chang Y, Meng FY, Wang MY, Xie QH, Tang F, Li PY, Song YH and Lin JS: MicroRNA-375 targets AEG-1 in hepatocellular carcinoma and suppresses liver cancer cell growth in vitro and in vivo. Oncogene 31: 3357-3369, 2012.

21. Mathé EA, Nguyen GH, Bowman ED, Zhao Y, Budhu A, Schetter AJ, Braun R, Reimers M, Kumamoto K, Hughes D, et al: MicroRNA expression in squamous cell carcinoma and adenocarcinoma of the esophagus: Associations with survival. Clin Cancer Res 15: 6192-6200, 2009.

22. Kano M, Seki N, Kikkawa N, Fujimura L, Hoshino I, Akutsu Y, Chiyomaru T, Enokida $\mathrm{H}$, Nakagawa $\mathrm{M}$ and Matsubara $\mathrm{H}$ : miR-145, miR-133a and miR-133b: Tumor-suppressive miRNAs target FSCN1 in esophageal squamous cell carcinoma. Int J Cancer 127: 2804-2814, 2010.

23. Kang DC, Su ZZ, Sarkar D, Emdad L, Volsky DJ and Fisher PB: Cloning and characterization of HIV-1-inducible astrocyte elevated gene-1, AEG-1. Gene 353: 8-15, 2005.

24. Brown DM and Ruoslahti E: Metadherin, a cell surface protein in breast tumors that mediates lung metastasis. Cancer Cell 5: 365-374, 2004

25. Li WF, Ou Q, Dai H and Liu CA: Lentiviral-mediated short hairpin RNA knockdown of MTDH inhibits cell growth and induces apoptosis by regulating the PTEN/AKT pathway in hepatocellular carcinoma. Int J Mol Sci 16: 19419-19432, 2015.

26. Yang C, Zheng S, Liu Q, Liu T, Lu M, Dai F, Gao X, Sheyhidin I and $\mathrm{Lu} \mathrm{X}$ : Metadherin is required for the proliferation, migration, and invasion of esophageal squamous cell carcinoma and its meta-analysis. Transl Res 166: 614-626.e2, 2015.
27. Holash J, Maisonpierre PC, Compton D, Boland P, Alexander CR, Zagzag D, Yancopoulos GD and Wiegand SJ: Vessel cooption, regression, and growth in tumors mediated by angiopoietins and VEGF. Science 284: 1994-1998, 1999.

28. Eriksson A, Cao R, Pawliuk R, Berg SM, Tsang M, Zhou D, Fleet C, Tritsaris K, Dissing S, Leboulch P and Cao Y: Placenta growth factor-1 antagonizes VEGF-C-induced angiogenesis and tumor growth by the formation of functionally inactive PIGF-1/VEGF heterodimers. Cancer Cell 1: 99-108, 2002.

29. Zhu GC, Yu CY, She L, Tan HL, Li G, Ren SL, Su ZW, Wei M, Huang DH, Tian YQ, et al: Metadherin regulation of vascular endothelial growth factor expression is dependent upon the PI3K/Akt pathway in squamous cell carcinoma of the head and neck. Medicine (Baltimore) 94: e502, 2015.

30. Emdad L, Lee SG, Su ZZ, Jeon HY, Boukerche H, Sarkar D and Fisher PB: Astrocyte elevated gene-1 (AEG-1) functions as an oncogene and regulates angiogenesis. Proc Natl Acad Sci USA 106: 21300-21305, 2009.

31. Fu M, Wang C, Li Z, Sakamaki T and Pestell RG: Minireview: Cyclin D1: Normal and abnormal functions. Endocrinology 145: 5439-5447, 2004.

32. Li PP, Feng LL, Chen N, Ge XL, Lv X, Lu K, Ding M, Yuan D and Wang X: Metadherin contributes to the pathogenesis of chronic lymphocytic leukemia partially through Wnt/ $\beta$-catenin pathway. Med Oncol 32: 479, 2015.

33. Cano A, Pérez-Moreno MA, Rodrigo I, Locascio A, Blanco MJ, del Barrio MG, Portillo F and Nieto MA: The transcription factor snail controls epithelial-mesenchymal transitions by repressing E-cadherin expression. Nat Cell Biol 2: 76-83, 2000

34. Li X, Kong X, Huo Q, Guo H, Yan S, Yuan C, Moran MS, Shao C and Yang Q: Metadherin enhances the invasiveness of breast cancer cells by inducing epithelial to mesenchymal transition. Cancer Sci 102: 1151-1157, 2011. 